\title{
A linear process algebraic format for probabilistic systems with data
}

\author{
Joost-Pieter Katoen ${ }^{\dagger *}$, Jaco van de Pol*, Mariëlle Stoelinga* and Mark Timmer* \\ *FMT Group, University of Twente, The Netherlands \\ \{vdpol,marielle,timmer\}@cs.utwente.nl \\ ${ }^{\dagger}$ MOVES Group, RWTH Aachen University, Germany
}

\begin{abstract}
This paper presents a novel linear process algebraic format for probabilistic automata. The key ingredient is a symbolic transformation of probabilistic process algebra terms that incorporate data into this linear format while preserving strong probabilistic bisimulation. This generalises similar techniques for traditional process algebras with data, and - more importantly - treats data and data-dependent probabilistic choice in a fully symbolic manner, paving the way to the symbolic analysis of parameterised probabilistic systems.
\end{abstract}

Keywords-probabilistic process algebra, linearisation, datadependent probabilistic choice, symbolic transformations

\section{INTRODUCTION}

Efficient model-checking algorithms exist, supported by powerful software tools, for verifying qualitative and quantitative properties for a wide range of probabilistic models. These techniques are applied in areas like security, randomised distributed algorithms, systems biology, and dependability and performance analysis. Major deficiencies of probabilistic model checking are the state explosion problem and the restricted treatment of data.

As opposed to process calculi like $\mu \mathrm{CRL}$ [1] and ELOTOS that support rich data types, the treatment of data in modeling formalisms for probabilistic systems is mostly neglected. Instead, the focus has been on understanding random phenomena and modeling the interplay between randomness and nondeterminism. Data is treated in a restricted manner: probabilistic process algebras typically allow a random choice over a fixed distribution, and input languages for model checkers such as the reactive module language of PRISM [2] or the probabilistic variant of Promela [3] only support basic data types, but neither support more advanced data structures or parameterised, i.e., state-dependent, random choice. To model realistic systems, however, convenient means for data modeling are indispensable.

Although parameterised probabilistic choice is semantically well-defined [4], the incorporation of data yields a significant increase of, or even an infinite, state space. Applying aggressive abstraction techniques for probabilistic models (e.g., [5], [6], [7], [8], [9]) obtain smaller models at the model level, but the successful analysis of data requires

This research has been partially funded by NWO under grant 612.063 .817 (SYRUP) and grant Dn 63-257 (ROCKS), and by the European Union under FP7-ICT-2007-1 grant 214755 (QUASIMODO). symbolic reduction techniques. These minimise stochastic models by syntactic transformations at the language level in order to minimise state spaces prior to their generation, while preserving functional and quantitative properties. Other approaches that partially deal with data are probabilistic CEGAR ([10], [11]) and the probabilistic GCL [12].

Our aim is to develop symbolic minimisation techniques - operating at the syntax level — for data-dependent probabilistic systems. The starting point for our work is laid down in this paper. We define a probabilistic variant of the process algebraic $\mu \mathrm{CRL}$ language [1], named prCRL, which treats data as first-class citizens. The language prCRL contains a carefully chosen minimal set of basic operators, on top of which syntactic sugar can be defined easily, and allows data-dependent probabilistic branching. To enable symbolic reductions, we provide a two-phase algorithm to transform prCRL terms into LPPEs: a probabilistic variant of linear process equations (LPEs) [13], which is a restricted form of process equations akin to the Greibach normal form for string grammars. We prove that our transformation is correct, in the sense that it preserves strong probabilistic bisimulation [14]. Similar linearisations have been provided for plain $\mu \mathrm{CRL}$ [15] and a real-time variant thereof [16].

To motivate the expected advantage of a probabilistic linear format, we draw an analogy with the purely functional case. There, LPEs provided a uniform and simple format for a process algebra with data. As a consequence of this simplicity, the LPE format was essential for theory development and tool construction. It lead to elegant proof methods, like the use of invariants for process algebra [13], and the cones and foci method for proof checking process equivalence ([17], [18]). It also enabled the application of model checking techniques to process algebra, such as optimisations from static analysis [19] (including dead variable reduction [20]), data abstraction [21], distributed model checking [22], symbolic model checking (either with BDDs [23] or by constructing the product of an LPE and a parameterised $\mu$-calculus formula ([24], [25])), and confluence reduction [26], a form of partial-order reduction.

In all these cases, the LPE format enabled a smooth theoretical development with rigorous correctness proofs (often checked in PVS), and a unifying tool implementation, enabling the cross-fertilisation of the various techniques by composing them as LPE-LPE transformations. 


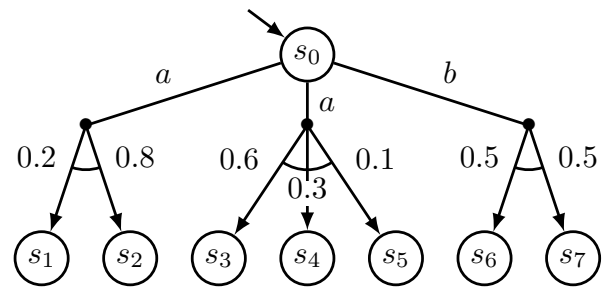

Figure 1. A probabilistic automaton.

To demonstrate the whole process of going from prCRL to LPPE and applying reductions to this LPPE, we discuss a case study of a leader election protocol.

We refer the reader to [27] for the extending version of the current paper, which includes proofs of all theorems and propositions and more details about the case study.

\section{PRELIMINARIES}

Let $\mathscr{P}(S)$ denote the powerset of the set $S$, i.e., the set of all its subsets, and let $\operatorname{Distr}(S)$ denote the set of all probability distributions over $S$, i.e., all functions $\mu: S \rightarrow[0,1]$ such that $\sum_{s \in S} \mu(s)=1$. If $S^{\prime} \subseteq S$, let $\mu\left(S^{\prime}\right)$ denote $\sum_{s \in S^{\prime}} \mu(s)$. For the injective function $f: S \rightarrow T$, let $\mu_{f} \in \operatorname{Distr}(T)$ such that $\mu_{f}(f(s))=\mu(s)$ for all $s \in S$. We use $\{*\}$ to denote a singleton set with a dummy element, and denote vectors and sets of vectors in bold.

\section{A. Probabilistic automata}

Probabilistic automata (PAs) are similar to labelled transition systems (LTSs), except that the transition function relates a state to a set of pairs of actions and distribution functions over successor states [28].

Definition 1. A probabilistic automaton (PA) is a tuple $\mathcal{A}=$ $\left\langle S, s^{0}, A, \Delta\right\rangle$, where

- $S$ is a finite set of states, of which $s^{0}$ is initial;

- $A$ is a finite set of actions;

- $\Delta: S \rightarrow \mathscr{P}(A \times \operatorname{Distr}(S))$ is a transition function. When $(a, \mu) \in \Delta(s)$, we write $s \stackrel{a}{\rightarrow} \mu$. This means that from state $s$ the action a can be executed, after which the probability to go to $s^{\prime} \in S$ equals $\mu\left(s^{\prime}\right)$.

Example 1. Figure 1 shows an example PA. Observe the nondeterministic choice between actions, after which the next state is determined probabilistically. Note that the same action can occur multiple times, each time with a different distribution to determine the next state. For this PA we have $s_{0} \stackrel{a}{\rightarrow} \mu$, where $\mu\left(s_{1}\right)=0.2$ and $\mu\left(s_{2}\right)=0.8$, and $\mu\left(s_{i}\right)=0$ for all other states $s_{i}$. Also, $s_{0} \stackrel{a}{\rightarrow} \mu^{\prime}$ and $s_{0} \stackrel{b}{\rightarrow} \mu^{\prime \prime}$, where $\mu^{\prime}$ and $\mu^{\prime \prime}$ can be obtained similarly.

\section{B. Strong probabilistic bisimulation}

Strong probabilistic bisimulation [14] is a probabilistic extension of the traditional notion of bisimulation introduced by Milner [29], equating any two processes that cannot be distinguished by an observer. It is well-known that strongly (probabilistic) bisimilar processes satisfy the same properties, as for instance expressed in $\mathrm{CTL}^{*}$ or $\mu$-calculus. Two states $s$ and $t$ of a $\mathrm{PA} \mathcal{A}$ are strongly probabilistic bisimilar (which we denote by $s \approx t$ ) if there exists an equivalence relation $R \subseteq S_{\mathcal{A}} \times S_{\mathcal{A}}$ such that $(s, t) \in R$, and

- if $(p, q) \in R$ and $p \stackrel{a}{\rightarrow} \mu$, there is a transition $q \stackrel{a}{\rightarrow} \mu^{\prime}$ such that $\mu \sim_{R} \mu^{\prime}$,

where $\mu \sim_{R} \mu^{\prime}$ is defined as $\forall C . \mu(C)=\mu^{\prime}(C)$, with $C$ ranging over the equivalence classes of states modulo $R$.

\section{Isomorphism}

Two states $s$ and $t$ of a PA $\mathcal{A}$ are isomorphic (which we denote by $s \equiv t$ ) if there exists a bijection $f: S_{\mathcal{A}} \rightarrow S_{\mathcal{A}}$ such that $f(s)=t$ and $\forall s^{\prime} \in S_{\mathcal{A}}, \mu \in \operatorname{Distr}\left(S_{\mathcal{A}}\right), a \in A_{\mathcal{A}}$. $s^{\prime} \stackrel{a}{\rightarrow} \mu \Leftrightarrow f\left(s^{\prime}\right) \stackrel{a}{\rightarrow} \mu_{f}$. Obviously, isomorphism implies strong probabilistic bisimulation.

\section{A PROCESS ALGEBRA INCORPORATING PROBABILISTIC CHOICE}

\section{A. The language prCRL}

We add a probabilistic choice operator to a restriction of full $\mu \mathrm{CRL}$ [1], obtaining a language called prCRL. We assume an external mechanism for the evaluation of expressions (e.g., equational logic), able to handle at least boolean expressions and real-valued expressions. Also, we assume that all closed expressions can be, and always are, evaluated. Note that this restricts the expressiveness of the data language. Let Act be a countable set of actions.

Definition 2. A process term in prCRL is any term that can be generated by the following grammar.

$$
p::=Y(\boldsymbol{t})|c \Rightarrow p| p+p\left|\sum_{x: D} p\right| a(\boldsymbol{t}) \sum_{x: D} f: p
$$

Here, $Y$ is a process name, $c$ a boolean expression, $a \in$ Act a (parameterised) atomic action, $f$ a real-valued expression yielding values in $[0,1]$ (further restricted below), $\boldsymbol{t}$ a vector of expressions, and $x$ a variable ranging over type $D$. We write $p=p^{\prime}$ for syntactically identical process terms.

$A$ process equation is an equation of the form $X(\boldsymbol{g}: \boldsymbol{G})=p$, where $\boldsymbol{g}$ is a vector of global variables and $G$ a vector of their types, and $p$ is a process term in which all free variables are elements of $\boldsymbol{g} ; X(\boldsymbol{g}: \boldsymbol{G})$ is called $a$ process with process name $X$ and right-hand side $p$. To obtain unique solutions, indirect (or direct) unguarded recursion is not allowed. Moreover, every construct $\sum_{x: D} f$ in a right-hand side $p$ should comply to $\sum_{d \in D} f[x:=d]=1$ for every possible valuation of the variables in $p$ (the summation now used in the mathematical sense). A prCRL specification is a set of process equations $X_{i}\left(\boldsymbol{g}_{\boldsymbol{i}}: \boldsymbol{G}_{\boldsymbol{i}}\right)=p_{i}$ such that all $X_{i}$ are named differently, and for every process instantiation $Y(\boldsymbol{t})$ occurring in some $p_{i}$ there exists a process equation $Y\left(\boldsymbol{g}_{\boldsymbol{i}}: \boldsymbol{G}_{\boldsymbol{i}}\right)=p_{i}$ such that $\boldsymbol{t}$ is of type $\boldsymbol{G}_{\boldsymbol{i}}$. 


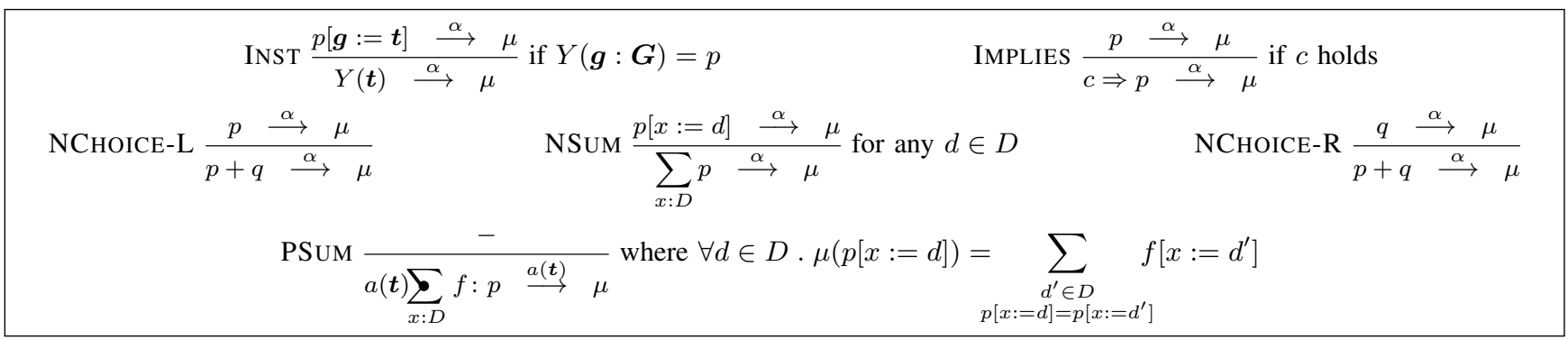

The initial process of a specification $P$ is an instantiation $Y(\boldsymbol{t})$ such that there exists an equation $Y(\boldsymbol{g}: \boldsymbol{G})=p$ in $P$, $\boldsymbol{t}$ is of type $\boldsymbol{G}$, and $Y(\boldsymbol{t})$ does not contain any free variables.

In a process term, $Y(\boldsymbol{t})$ denotes process instantiation (allowing recursion). The term $c \Rightarrow p$ is equal to $p$ if the condition $c$ holds, and cannot do anything otherwise. The + operator denotes nondeterministic choice, and $\sum_{x: D} p$ a (possibly infinite) nondeterministic choice over data type $D$. Finally, $a(\boldsymbol{t}) \sum_{x: D} f: p$ performs the action $a(\boldsymbol{t})$ and then does a probabilistic choice over $D$. It uses the value $f[x:=d]$ as the probability of choosing each $d \in D$. We do not consider process terms of the form $p \cdot p$ (where - denotes sequential composition), as this significantly increases the difficulty of both linearisation and optimisation [16]. Moreover, most specifications used in practice can be written without this form.

The operational semantics of prCRL is given in terms of PAs. The states are process terms, the initial state is the initial process, the action set is Act, and the transition relation is the smallest relation satisfying the SOS rules in Table I. Here, $p[x:=d]$ is used to denote the substitution of all occurrences of $x$ in $p$ by $d$. Similarly, $p[\boldsymbol{t}:=\boldsymbol{x}]$ is used to denote $p[\boldsymbol{t}(1):=\boldsymbol{x}(1)] \cdots[\boldsymbol{t}(n):=\boldsymbol{x}(n)]$. For brevity, we use $\alpha$ to denote an action name together with its parameters. A mapping to PAs is only provided for processes without any free variables; as Definition 2 only allows such processes, this imposes no further restrictions.

Proposition 1. The SOS-rule PSUM defines a probability distribution $\mu$.

Example 2. The following process equation models a system that continuously writes data elements of the finite type $D$ randomly. After each write, it beeps with probability 0.1. Recall that $\{*\}$ denotes a singleton set with an anonymous element. We use it here since the probabilistic choice is trivial and the value of $j$ is never used. For brevity, we abuse notation by interpreting a process equation as a specification.

$$
\begin{gathered}
B=\tau \sum_{d: D} \frac{1}{|D|}: \operatorname{send}(d) \sum_{i:\{1,2\}} \quad(\text { if } i=1 \text { then } 0.1 \text { else } 0.9): \\
+\left(i=1 \Rightarrow \operatorname{beep}() \sum_{j:\{*\}} 1.0: B\right) \\
+(i \neq 1 \Rightarrow B))
\end{gathered}
$$

\section{B. Syntactic sugar}

For notational ease we define some syntactic sugar. Let $a$ be an action, $\boldsymbol{t}$ an expression vector, $c$ a condition, and $p, q$ two process terms. Then,

$$
\begin{array}{lll}
a & \stackrel{\text { def }}{=} & a() \\
p \triangleleft c \triangleright q & \stackrel{\text { def }}{=} & (c \Rightarrow p)+(\neg c \Rightarrow q) \\
a(\boldsymbol{t}) \cdot p & \stackrel{\text { def }}{=} & a(\boldsymbol{t}) \sum_{x:\{*\}} 1.0: p \\
a(\boldsymbol{t}) U_{d: D} c \Rightarrow p & \stackrel{\text { def }}{=} & a(\boldsymbol{t}) \sum_{d: D} f: p
\end{array}
$$

where $x$ does not occur in $p$ and $f$ is the function 'if $c$ then $\frac{1}{|\{e \in D \mid c[d:=e]\}|}$ else 0 '. Note that $U_{d: D} c \Rightarrow p$ is the uniform choice among a set, choosing only from its elements that fulfil a certain condition.

For finite probabilistic sums that do not depend on data,

$$
a(\boldsymbol{t})\left(u_{1}: p_{1} \oplus u_{2}: p_{2} \oplus \cdots \oplus u_{n}: p_{n}\right)
$$

is used to abbreviate $a(\boldsymbol{t}) \sum_{x:\{1, \ldots, n\}} f: p$ with $f[x:=i]=$ $u_{i}$ and $p[x:=i]=p_{i}$ for all $1 \leq i \leq n$.

Example 3. The process equation of Example 2 can now be represented as follows:

$$
B=\tau \sum_{d: D} \frac{1}{|D|}: \operatorname{send}(d)(0.1: \text { beep } \cdot B \oplus 0.9: B)
$$

Example 4. Let $X$ continuously send an arbitrary element of some type $D$ that is contained in a finite set $\operatorname{Set}_{D}$, according to a uniform distribution. It is represented by

$$
X\left(s: \operatorname{Set}_{D}\right)=\tau \bigcup_{d: D} \operatorname{contains}(s, d) \Rightarrow \operatorname{send}(d) \cdot X(s),
$$

where contains $(s, d)$ is assumed to hold when $s$ contains $d$.

\section{A LINEAR FORMAT FOR PRCRL}

\section{A. The LPE and LPPE formats}

In the non-probabilistic setting, LPEs are given by the following equation [16]:

$$
X(\boldsymbol{g}: \boldsymbol{G})=\sum_{i \in I} \sum_{\boldsymbol{d}_{\boldsymbol{i}}: \boldsymbol{D}_{\boldsymbol{i}}} c_{i} \Rightarrow a_{i}\left(b_{i}\right) \cdot X\left(\boldsymbol{n}_{\boldsymbol{i}}\right),
$$

where $G$ is a type for state vectors (containing the global variables), $I$ a set of summand indices, and $D_{i}$ a type for local variable vectors for summand $i$. The summations 
represent nondeterministic choices; the outer between different summands, the inner between different possibilities for the local variables. Furthermore, each summand $i$ has an action $a_{i}$ and three expressions that may depend on the state $\boldsymbol{g}$ and the local variables $\boldsymbol{d}_{\boldsymbol{i}}$ : the enabling condition $c_{i}$, the action-parameter vector $b_{i}$, and the next-state vector $\boldsymbol{n}_{\boldsymbol{i}}$. The initial process $X\left(\boldsymbol{g}^{0}\right)$ is represented by the initial vector $\boldsymbol{g}^{0}$, and $g^{0}$ is used to denote the initial value of global variable $g$.

Example 5. Consider a system consisting of two buffers, $B_{1}$ and $B_{2}$. Buffer $B_{1}$ reads a message of type $D$ from the environment, and sends it synchronously to $B_{2}$. Then, $B_{2}$ writes the message. The following LPE has exactly this behaviour when initialised with $a=1$ and $b=1$.

$$
\begin{aligned}
& X(a:\{1,2\}, b:\{1,2\}, x: D, y: D)= \\
& \sum_{d: D} \quad a=1 \quad \Rightarrow \operatorname{read}(d) \cdot X(2, b, d, y) \\
& +\quad a=2 \wedge b=1 \Rightarrow \operatorname{comm}(x) \cdot X(1,2, x, x) \\
& +\quad b=2 \quad \Rightarrow \operatorname{write}(y) \cdot X(a, 1, x, y)
\end{aligned}
$$

Note that the first summand models $B_{1}$ 's reading, the second the inter-buffer communication, and the third $B_{2}$ 's writing. The global variables $a$ and $b$ are used as program counters for $B_{1}$ and $B_{2}$, and $x$ and $y$ for their local memory.

As our intention is to develop a linear format for prCRL that can easily be mapped onto PAs, it should follow the concept of nondeterministically choosing an action and probabilistically determining the next state. Therefore, a natural adaptation is the format given by the following definition.

Definition 3. An LPPE (linear probabilistic process equation) is a prCRL specification consisting of one process equation, of the following format:

$$
X(\boldsymbol{g}: \boldsymbol{G})=\sum_{i \in I} \sum_{\boldsymbol{d}_{\boldsymbol{i}}: \boldsymbol{D}_{\boldsymbol{i}}} c_{i} \Rightarrow a_{i}\left(b_{i}\right) \sum_{\boldsymbol{e}_{\boldsymbol{i}}: \boldsymbol{E}_{\boldsymbol{i}}} f_{i}: X\left(\boldsymbol{n}_{\boldsymbol{i}}\right)
$$

Compared to the LPE we added a probabilistic choice over an additional vector of local variables $\boldsymbol{e}_{\boldsymbol{i}}$. The corresponding probability distribution expression $f_{i}$, as well as the nextstate vector $\boldsymbol{n}_{\boldsymbol{i}}$, can now also depend on $\boldsymbol{e}_{\boldsymbol{i}}$.

\section{B. Operational semantics}

As the behaviour of an LPPE is uniquely determined by its global variables, the states of the underlying PA are precisely all vectors $\boldsymbol{g}^{\prime} \in \boldsymbol{G}$ (with the initial vector as initial state). From the SOS rules it follows that for all $\boldsymbol{g}^{\prime} \in \boldsymbol{G}$, there is a transition $\boldsymbol{g}^{\prime} \stackrel{a(\boldsymbol{q})}{\rightarrow} \mu$ if and only if for at least one summand $i$ there is a choice of local variables $d_{i}^{\prime} \in D_{i}$ such that

$$
\begin{aligned}
& c_{i}\left(\boldsymbol{g}^{\prime}, \boldsymbol{d}_{\boldsymbol{i}}^{\prime}\right) \wedge a_{i}\left(b_{i}\left(\boldsymbol{g}^{\prime}, \boldsymbol{d}_{\boldsymbol{i}}^{\prime}\right)\right)=a(\boldsymbol{q}) \wedge \forall \boldsymbol{e}_{\boldsymbol{i}}^{\prime} \in \boldsymbol{E}_{\boldsymbol{i}} . \\
& \mu\left(n_{i}\left(\boldsymbol{g}^{\prime}, \boldsymbol{d}_{\boldsymbol{i}}^{\prime}, \boldsymbol{e}_{\boldsymbol{i}}^{\prime}\right)\right)=\sum_{\substack{\boldsymbol{e}_{\boldsymbol{i}}^{\prime \prime} \in \boldsymbol{E}_{\boldsymbol{i}} \\
n_{i}\left(\boldsymbol{g}^{\prime}, \boldsymbol{d}_{\boldsymbol{i}}^{\prime}, \boldsymbol{e}_{\boldsymbol{i}}^{\prime}\right)=n_{i}\left(\boldsymbol{g}^{\prime}, \boldsymbol{d}_{\boldsymbol{i}}^{\prime}, \boldsymbol{e}_{\boldsymbol{i}}^{\prime \prime}\right)}} f_{i}\left(\boldsymbol{g}^{\prime}, \boldsymbol{d}_{\boldsymbol{i}}^{\prime}, \boldsymbol{e}_{\boldsymbol{i}}^{\prime \prime}\right),
\end{aligned}
$$

where for $c_{i}$ and $b_{i}$ the notation $\left(\boldsymbol{g}^{\prime}, \boldsymbol{d}_{\boldsymbol{i}}^{\prime}\right)$ is used to abbreviate $\left[\boldsymbol{g}:=\boldsymbol{g}^{\prime}, \boldsymbol{d}_{\boldsymbol{i}}:=\boldsymbol{d}_{\boldsymbol{i}}^{\prime}\right]$, and for $n_{i}$ and $f_{i}$ we use $\left(\boldsymbol{g}^{\prime}, \boldsymbol{d}_{\boldsymbol{i}}^{\prime}, \boldsymbol{e}_{\boldsymbol{i}}^{\prime}\right)$ to abbreviate $\left[g:=g^{\prime}, d_{i}:=d_{i}^{\prime}, e_{i}:=e_{i}^{\prime}\right]$.
Example 6. Consider a system that continuously sends a random element of a finite type $D$. It is represented by

$$
X=\tau \sum_{d: D} \frac{1}{|D|}: \operatorname{send}(d) \cdot X,
$$

and is easily seen to be isomorphic to the following LPPE when initialised with $p c=1$. The initial value $d^{0}$ can be chosen arbitrarily, as it will be overwritten before used.

$$
\begin{aligned}
X(p c: & \{1,2\}, d: D)= \\
& p c=1 \Rightarrow \tau \sum_{d: D} \frac{1}{|D|}: X(2, d) \\
+ & p c=2 \Rightarrow \operatorname{send}(d) \sum_{y:\{*\}} 1.0: X\left(1, d^{0}\right)
\end{aligned}
$$

Obviously, the earlier defined syntactic sugar could also be used on LPPEs, writing $\operatorname{send}(d) \cdot X\left(1, d^{0}\right)$ in the second summand. However, as linearisation will be defined only on the basic operators, we will often keep writing the full form.

\section{LINEARISATION}

Linearisation of a prCRL specification is performed in two steps: (1) Every right-hand side becomes a summation of process terms, each of which contains exactly one action; this is the intermediate regular form (IRF). This step is performed by Algorithm 1, which uses Algorithms 2 and 3. (2) An LPPE is created based on the IRF, using Algorithm 4. The algorithms are shown in detail on the following pages. We first illustrate both steps based on two examples.

Example 7. Consider the specification $X=a \cdot b \cdot c \cdot X$. We can transform $X$ into the IRF $\left\{X_{1}=a \cdot X_{2}, X_{2}=b \cdot X_{3}\right.$, $\left.X_{3}=c \cdot X_{1}\right\}$ (with initial process $X_{1}$ ). Now, a strongly bisimilar (in this case even isomorphic) LPPE can be constructed by introducing a program counter $p c$ that keeps track of the subprocess that is currently active, as below. It is not hard to see that $Y(1)$ generates the same state space as $X$.

$$
\begin{aligned}
Y(p c:\{1,2,3\})=p c=1 & \Rightarrow a \cdot Y(2) \\
+p c=2 & \Rightarrow b \cdot Y(3) \\
+p c=3 & \Rightarrow c \cdot Y(1)
\end{aligned}
$$

Example 8. Now consider the following specification, consisting of two process equations with parameters. Let $B\left(d^{\prime}\right)$ be the initial process for some $d^{\prime} \in D$.

$$
\begin{aligned}
& B(d: D)= \\
& \tau \sum_{e: E} \frac{1}{|E|}: \operatorname{send}(d+e) \sum_{i:\{1,2\}}(\text { if } i=1 \text { then } 0.1 \text { else } 0.9): \\
& \quad\left(\left(i=1 \Rightarrow \operatorname{crash} \sum_{j:\{*\}} 1.0: B(d)\right)+(i \neq 1 \Rightarrow C(d+1))\right) \\
& C(f: D)= \\
& \quad \text { write }\left(f^{2}\right) \sum_{k:\{*\}} 1.0: \sum_{g: D} \operatorname{write}(f+g) \sum_{l:\{*\}} 1.0: B(f+g)
\end{aligned}
$$


Again we introduce a new process for each subprocess. For brevity we use $(\boldsymbol{p})$ for $(d: D, f: D, e: E, i:\{1,2\}, g: D)$. The initial process is $X_{1}\left(d^{\prime}, f^{0}, e^{0}, i^{0}, g^{0}\right)$, where $f^{0}, e^{0}, i^{0}$, and $g^{0}$ can be chosen arbitrarily.

$$
\begin{aligned}
& X_{1}(\boldsymbol{p})= \tau \sum_{e: E} \frac{1}{|E|}: X_{2}\left(d, f^{0}, e, i^{0}, g^{0}\right) \\
& X_{2}(\boldsymbol{p})= \operatorname{send}(d+e) \sum_{i:\{1,2\}} \quad(\text { if } i=1 \text { then } 0.1 \text { else } 0.9): \\
& X_{3}\left(d, f^{0}, e^{0}, i, g^{0}\right) \\
& X_{3}(\boldsymbol{p})=\left(i=1 \Rightarrow \operatorname{crash} \sum_{j:\{*\}} 1.0: X_{1}\left(d, f^{0}, e^{0}, i^{0}, g^{0}\right)\right) \\
&+\left(i \neq 1 \Rightarrow \operatorname{write}\left((d+1)^{2}\right) \sum_{k:\{*\}} 1.0:\right. \\
&\left.X_{4}\left(d^{\prime}, d+1, e^{0}, i^{0}, g^{0}\right)\right) \\
& X_{4}(\boldsymbol{p})= \sum_{g: D} \operatorname{write}(f+g) \sum_{l:\{*\}} 1.0: X_{1}\left(f+g, f^{0}, e^{0}, i^{0}, g^{0}\right)
\end{aligned}
$$

Note that we added global variables to remember the values of variables that were bound by a nondeterministic or probabilistic summation. As the index variables $j, k$ and $l$ are never used, they are not remembered. We also reset variables that are not syntactically used in their scope to keep the state space minimal.

Again, the LPPE is obtained by introducing a program counter. The initial vector is $\left(1, d^{\prime}, f^{0}, e^{0}, i^{0}, g^{0}\right)$, and $f^{0}, e^{0}, i^{0}$, and $g^{0}$ can again be chosen arbitrarily.

$$
\begin{aligned}
& X(p c:\{1,2,3,4\}, d: D, f: D, e: E, i:\{1,2\}, g: D)= \\
& p c=1 \Rightarrow \tau \sum_{e: E} \frac{1}{|E|}: X\left(2, d, f^{0}, e, i^{0}, g^{0}\right) \\
& +p c=2 \Rightarrow \operatorname{send}(d+e) \sum_{i:\{1,2\}}(\text { if } i=1 \text { then } 0.1 \text { else } 0.9): \\
& +p c=3 \wedge i=1 \Rightarrow \operatorname{crash} \sum_{j:\{*\}} 1.0: X\left(1, d, f^{0}, e^{0}, i, g^{0}\right) \\
& \left.+p c=3 \wedge i \neq 1 \Rightarrow e^{0}, i^{0}, g^{0}\right) \\
& \quad \text { write }\left((d+1)^{2}\right) \sum_{k:\{*\}} 1.0: X\left(4, d^{0}, d+1, e^{0}, i^{0}, g^{0}\right) \\
& +\sum_{g: D} p c=4 \Rightarrow \operatorname{write}(f+g) \sum_{l:\{*\}} 1.0: X\left(1, f+g, f^{0}, e^{0}, i^{0}, g^{0}\right)
\end{aligned}
$$

\section{A. Transforming from prCRL to IRF}

We now formally define the IRF, and then discuss the transformation from prCRL to IRF in more detail.

Definition 4. A process term is in IRF if it adheres to the following grammar:

$$
p::=c \Rightarrow p|p+p| \sum_{x: D} p \mid a(\boldsymbol{t}) \sum_{x: D} f: Y(\boldsymbol{t})
$$

Note that in IRF every probabilistic sum goes to a process instantiation, and that process instantiations do not occur in any other way. A process equation is in IRF if its righthand side is in IRF, and a specification is in IRF if all its process equations are in IRF and all its processes have the same global variables. For every specification $P$ with initial process $X(\boldsymbol{g})$ there exists a specification $P^{\prime}$ in IRF with initial process $X^{\prime}\left(\boldsymbol{g}^{\prime}\right)$ such that $X(\boldsymbol{g}) \approx X^{\prime}\left(\boldsymbol{g}^{\prime}\right)$ (as we provide an algorithm to find it). However, it is not hard to see that $P^{\prime}$ is not unique. Also, not necessarily $X(\boldsymbol{g}) \equiv X^{\prime}\left(\boldsymbol{g}^{\prime}\right)$.

Clearly, every specification $P$ representing a finite PA can be transformed to an IRF describing an isomorphic PA: just define a data type $S$ with an element $s_{i}$ for every state of the PA underlying $P$, and create a process $X(s: S)$ consisting of a summation of terms of the form $s=s_{i} \Rightarrow a(\boldsymbol{t})\left(p_{1}: s_{1} \oplus p_{2}: s_{2} \ldots \oplus p_{n}: s_{n}\right)$ (one for each transition $s_{i} \stackrel{a(\boldsymbol{t})}{\rightarrow} \mu$, where $\mu\left(s_{1}\right)=p_{1}, \mu\left(s_{2}\right)=$ $\left.p_{2}, \ldots, \mu\left(s_{n}\right)=p_{n}\right)$. However, this transformation completely defeats its purpose, as the whole idea behind the LPPE is to apply reductions before having to compute all states of the original specification.

\section{Overview of the transformation to IRF.}

Algorithm 1 transforms a specification $P$ with initial process $X_{1}(\boldsymbol{g})$ to a specification $P^{\prime}$ with initial process $X_{1}^{\prime}\left(\boldsymbol{g}^{\prime}\right)$, such that $X_{1}(\boldsymbol{g}) \approx X_{1}^{\prime}\left(\boldsymbol{g}^{\prime}\right)$ and $P^{\prime}$ is in IRF. It requires that all global and local variables of $P$ have unique names (which is easily achieved by $\alpha$-conversion). Three important variables are used: (1) done is a set of process equations that are already in IRF; (2) toTransform is a set of process equations that still have to be transformed to IRF;

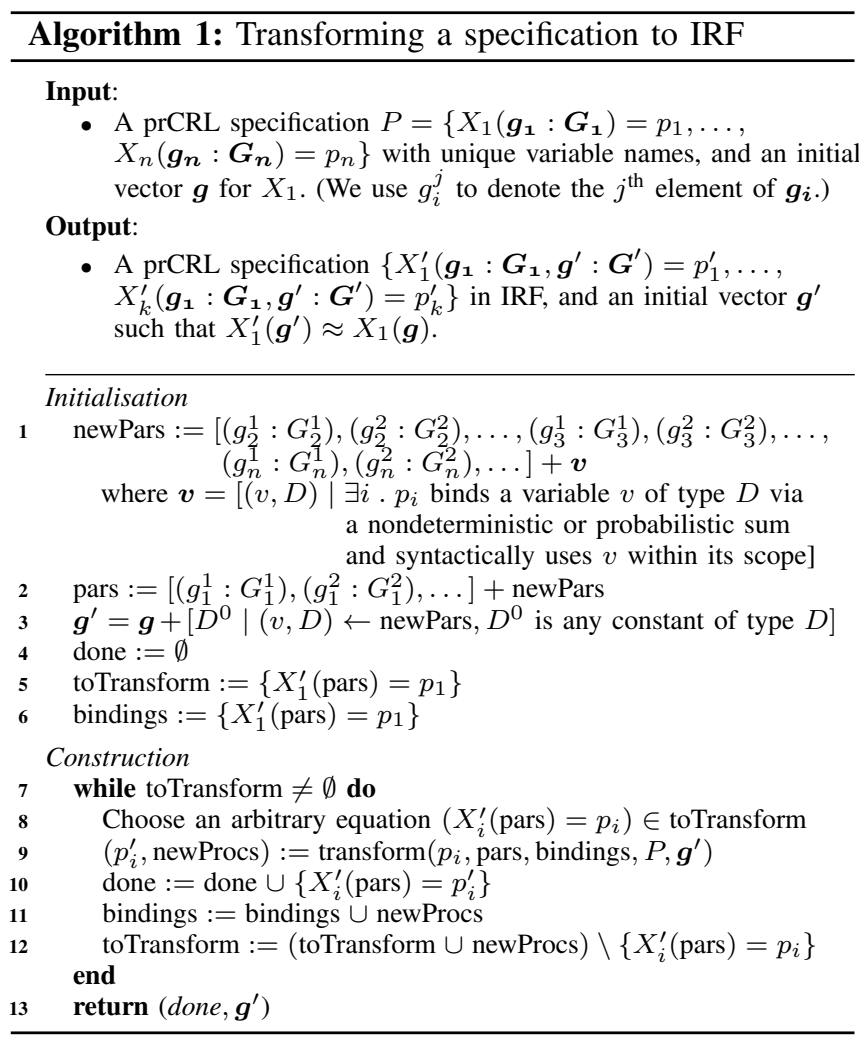



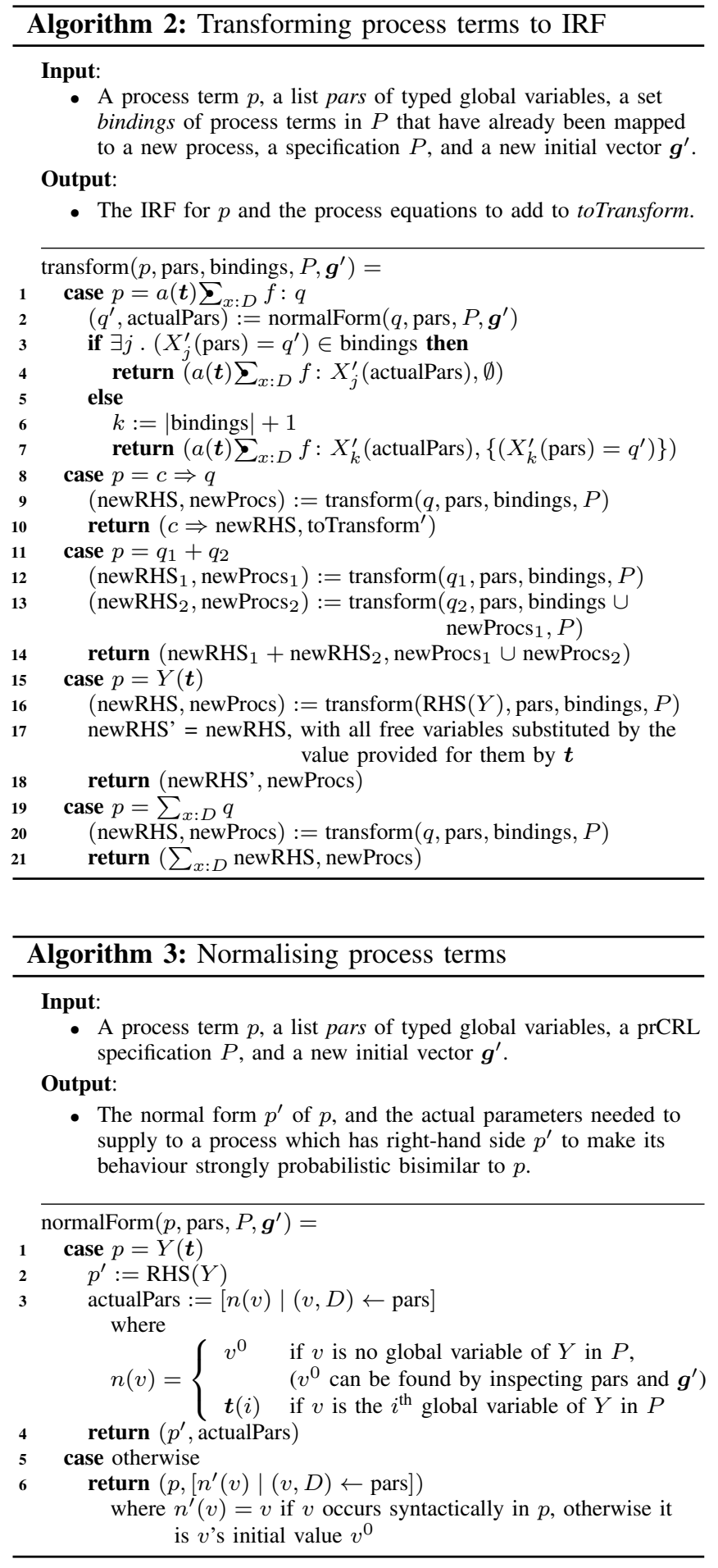

(3) bindings is a set of process equations $X_{i}^{\prime}$ (pars) $=p_{i}$, such that $X_{i}^{\prime}$ (pars) is the process in done $\cup$ toTransform representing the process term $p_{i}$ of the original specification.

Initially, done is empty and we bind the right-hand side of the initial process to $X_{1}^{\prime}$ (and add this equation to toTransform). Also, pars becomes the list of all variables occurring in $P$ as global variables or in a summation (and syntactically used after being bound). The new initial vector is computed by appending dummy values to the original initial vector for the newly added parameters. (Haskell-like list comprehension is used to denote this formally.) Then, basically we repeatedly take a process equation $X_{i}^{\prime}($ pars $)=p_{i}$ from toTransform, transform $p_{i}$ to a strongly probabilistic bisimilar IRF $p_{i}^{\prime}$ using Algorithm 2, add the process $X_{i}^{\prime}($ pars $)=p_{i}^{\prime}$ to done, and remove $X_{i}^{\prime}($ pars $)=p_{i}$ from toTransform. The transformation may have introduced new processes, which are added to toTransform, and bindings is updated accordingly.

\section{Transforming single process terms to IRF.}

Algorithm 2 transforms process terms to IRF recursively by means of a case distinction over the structure of the terms.

The base case is a probabilistic choice $a(t) \sum_{x: D} f: q$. The corresponding IRF is $a(\boldsymbol{t}) \sum_{x: D} f: X_{i}^{\prime}$ (actualPars), where $X_{i}^{\prime}$ is either the process name already mapped to $q$ (as stored in bindings), or a new process name when there did not yet exist such a process. More precisely, instead of $q$ we use its normal form (computed by Algorithm 3); when $q$ is a process instantiation $Y(\boldsymbol{t})$, its normal form is the right-hand side of $Y$, otherwise it is just $q$. When $q$ is not a process instantiation, the actual parameters for $X_{i}^{\prime}$ are just the global variables (possibly resetting variables that are not used in $q)$. When $q=Y\left(v_{1}, v_{2}, \ldots, v_{n}\right)$, all global variables are reset, except the ones corresponding to the original global variables of $Y$; for them $v_{1}, v_{2}, \ldots, v_{n}$ are used. Newly created processes are added to toTransform.

For a summation $q_{1}+q_{2}$, the IRF is $q_{1}^{\prime}+q_{2}^{\prime}$ (with $q_{i}^{\prime}$ an IRF of $q_{i}$ ). For the condition $c \Rightarrow q_{1}$ it is $c \Rightarrow q_{1}^{\prime}$, and for $\sum_{x: D} q_{1}$ it is $\sum_{x: D} q_{1}^{\prime}$. Finally, the IRF for $Y(\boldsymbol{t})$ is the IRF for the right-hand side of $Y$, where the global variables of $Y$ occurring in this term have been substituted by the expressions given by $\boldsymbol{t}$.

Example 9. We linearise two example specifications: $P_{1}=\left\{X_{1}=a \cdot b \cdot c \cdot X_{1}+c \cdot X_{2}, X_{2}=a \cdot b \cdot c \cdot X_{1}\right\}$, and $P_{2}=\left\{X_{3}(d: D)=\sum_{e: D} a(d+e) \cdot c(e) \cdot X_{3}(5)\right\} \quad$ (with initial processes $X_{1}$ and $X_{3}\left(d^{\prime}\right)$ ). Tables II and III show done, bindings and toTransform at line 7 of Algorithm 1 for every iteration. As both bindings and done only grow, we just list their additions. For layout purposes, we omit the parameters $(d: D, e: D)$ of every $X_{i}^{\prime \prime}$ in Table III. The initial processes are $X_{1}^{\prime}$ and $X_{1}^{\prime \prime}\left(d^{\prime}, e^{0}\right)$ for some $e^{0} \in D$.

Theorem 1. Let $P=\left\{X_{1}\left(\boldsymbol{g}_{\mathbf{1}}: \boldsymbol{G}_{\mathbf{1}}\right)=p_{1}, \ldots\right.$, $\left.X_{n}\left(\boldsymbol{g}_{\boldsymbol{n}}: \boldsymbol{G}_{\boldsymbol{n}}\right)=p_{n}\right\}$ be a $\mathrm{prCRL}$ specification with initial vector $g$. Given these inputs Algorithm 1 terminates, and the specification $P^{\prime}=\left\{X_{1}^{\prime}\left(\boldsymbol{g}_{\mathbf{1}}: \boldsymbol{G}_{\mathbf{1}}, \boldsymbol{g}^{\prime}: \boldsymbol{G}^{\prime}\right)=p_{1}^{\prime}, \ldots\right.$, $\left.X_{k}^{\prime}\left(\boldsymbol{g}_{\mathbf{1}}: \boldsymbol{G}_{\mathbf{1}}, \boldsymbol{g}^{\prime}: \boldsymbol{G}^{\prime}\right)=p_{k}^{\prime}\right\}$ and initial vector $\boldsymbol{g}^{\prime}$ it returns are such that $X_{1}^{\prime}\left(\boldsymbol{g}^{\prime}\right)$ in $P^{\prime}$ is strongly probabilistic bisimilar to $X_{1}(g)$ in $P$. Also, $P^{\prime}$ is in IRF.

The following example shows that Algorithm 1 does not 
Table II

TRANSFORMing $\left\{X_{1}=a \cdot b \cdot c \cdot X_{1}+c \cdot X_{2}, X_{2}=a \cdot b \cdot c \cdot X_{1}\right\}$ With INITIAL PROCESS $X_{1}$ TO IRF.

\begin{tabular}{c|c|c|c} 
& done & toTransform & bindings \\
\hline 0 & $\emptyset$ & $X_{1}^{\prime}=a \cdot b \cdot c \cdot X_{1}+c \cdot X_{2}$ & $X_{1}^{\prime}=a \cdot b \cdot c \cdot X_{1}+c \cdot X_{2}$ \\
\hline 1 & $X_{1}^{\prime}=a \cdot X_{2}^{\prime}+c \cdot X_{3}^{\prime}$ & $X_{2}^{\prime}=b \cdot c \cdot X_{1}, X_{3}^{\prime}=a \cdot b \cdot c \cdot X_{1}$ & $X_{2}^{\prime}=b \cdot c \cdot X_{1}, X_{3}^{\prime}=a \cdot b \cdot c \cdot X_{1}$ \\
\hline 2 & $X_{2}^{\prime}=b \cdot X_{4}^{\prime}$ & $X_{3}^{\prime}=a \cdot b \cdot c \cdot X_{1}, X_{4}^{\prime}=c \cdot X_{1}$ & $X_{4}^{\prime}=c \cdot X_{1}$ \\
\hline 3 & $X_{3}^{\prime}=a \cdot X_{2}^{\prime}$ & $X_{4}^{\prime}=c \cdot X_{1}$ & \\
\hline 4 & $X_{4}^{\prime}=c \cdot X_{1}^{\prime}$ & $\emptyset$ &
\end{tabular}

Table III

TRANSFORMing $\left\{X_{3}(d: D)=\sum_{e: D} a(d+e) \cdot c(e) \cdot X_{3}(5)\right\}$ With INITIAL PROCESS $X_{3}\left(d^{\prime}\right)$ To IRF.

\begin{tabular}{c|c|c|c} 
& done & toTransform & bindings \\
\hline 0 & $\emptyset$ & $X_{1}^{\prime \prime}=\sum_{e: D} a(d+e) \cdot c(e) \cdot X_{3}(5)$ & $X_{1}^{\prime \prime}=\sum_{e: D} a(d+e) \cdot c(e) \cdot X_{3}(5)$ \\
\hline 1 & $X_{1}^{\prime \prime}=\sum_{e: D} a(d+e) \cdot X_{2}^{\prime \prime}\left(d^{\prime}, e\right)$ & $X_{2}^{\prime \prime}=c(e) \cdot X_{3}(5)$ & $X_{2}^{\prime \prime}=c(e) \cdot X_{3}(5)$ \\
\hline 2 & $X_{2}^{\prime \prime}=c(e) \cdot X_{1}^{\prime \prime}\left(5, e^{0}\right)$ & $\emptyset$ &
\end{tabular}

always compute an isomorphic specification.

Example 10. Let $X=\sum_{d: D} a(d) \cdot b(f(d)) \cdot X$, with $f(d)=0$ for all $d \in D$. Then, our procedure will yield the specification $\left\{X_{1}^{\prime}(d: D)=\sum_{d: D} a(d) \cdot X_{2}^{\prime}(d)\right.$, $\left.X_{2}^{\prime}(d: D)=b(f(d)) \cdot X_{1}^{\prime}\left(d^{0}\right)\right\}$ with initial process $X_{1}^{\prime}\left(d^{0}\right)$ for an arbitrary $d^{0} \in D$. Note that the number of states of $X_{1}^{\prime}\left(d^{0}\right)$ is $|D|+1$ for any $d^{0} \in D$. However, the state space of $X$ only consists of the two states $X$ and $b(0) \cdot X$.

\section{B. Transforming from IRF to LPPE}

Based on a specification $P^{\prime}$ in IRF, Algorithm 4 constructs an LPPE $X$. The global variables of $X$ are a newly introduced program counter $p c$ and all global variables of $P^{\prime}$. To construct the summands for $X$, the algo-

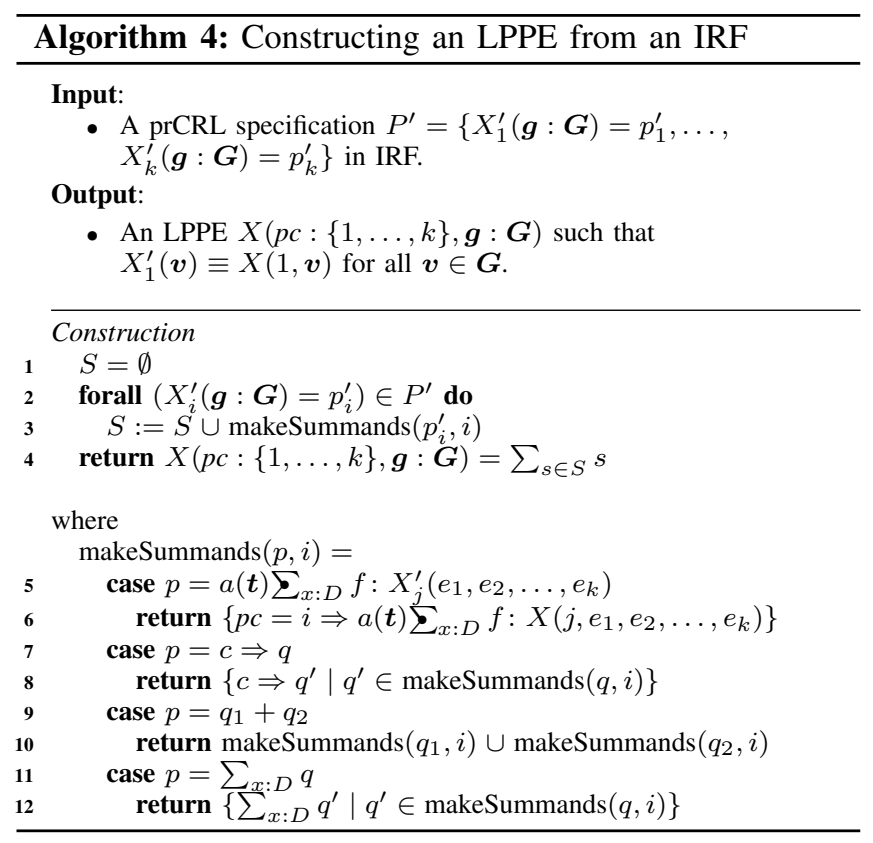

rithm ranges over the process equations in $P^{\prime}$. For each equation $X_{i}^{\prime}=a(\boldsymbol{t}) \sum_{x: D} f: X_{j}^{\prime}\left(e_{1}, \ldots, e_{k}\right)$, a summand $p c=i \Rightarrow a(\boldsymbol{t}) \sum_{x: D} f: X\left(j, e_{1}, \ldots, e_{k}\right)$ is constructed. For an equation $X_{i}^{\prime}=q_{1}+q_{2}$ the union of the summands produced by $X_{i}^{\prime}=q_{1}$ and $X_{i}^{\prime}=q_{2}$ is taken. For $X_{i}^{\prime}=c \Rightarrow q$ the condition $c$ is prefixed before the summands produced by $X_{i}^{\prime}=q$; the nondeterministic sum is handled similarly.

To be precise, in every summand of a specification obtained this way, the nondeterministic sums should still be moved to the front to obtain an actual LPPE. This does not change behaviour because of the assumed uniqueness of variable names. Moreover, separate nondeterministic sums and separate conditions should be merged (by using vectors and conjunctions, respectively).

Theorem 2. Let $P^{\prime}=\left\{X_{1}^{\prime}(\boldsymbol{g}: \boldsymbol{G})=p_{1}^{\prime}, \ldots, X_{k}^{\prime}(\boldsymbol{g}: \boldsymbol{G})=\right.$ $\left.p_{k}^{\prime}\right\}$ be a specification in IRF, and $X(p c:\{1, \ldots, k\}, \boldsymbol{g}: \boldsymbol{G})$ the LPPE obtained by applying Algorithm 4 to $P^{\prime}$. Then, $X_{1}^{\prime}(\boldsymbol{v}) \equiv X(1, \boldsymbol{v})$ for every $\boldsymbol{v} \in \boldsymbol{G}$. Also, $X$ is an LPPE (after, within each summand, moving the nondeterministic sums to the beginning and merging separate nondeterministic sums and separate conditions).

Proposition 2. The time complexity of linearising a specification $P$ is in $O\left(n^{3}\right)$, where $n=\sum_{\left(X_{i}\left(\boldsymbol{g}_{i}: \boldsymbol{G}_{i}\right)=p_{i}\right) \in P}$ size $\left(\boldsymbol{g}_{\boldsymbol{i}}\right)+\operatorname{size}\left(p_{i}\right)$. The LPPE size is in $O\left(n^{2}\right)$.

Although the transformation to LPPE increases the size of the specification, it facilitates optimisations to reduce the state space (which is worst-case in $O\left(2^{n}\right)$ ).

Example 11. Looking at the IRFs obtained in Example 9, it follows that $X_{1}^{\prime} \approx X(1)$ where $X(p c:\{1,2,3,4\})=$ $(p c=1 \Rightarrow a \cdot X(2))+(p c=1 \Rightarrow c \cdot X(3))+(p c=2 \Rightarrow$ $b \cdot X(4))+(p c=3 \Rightarrow a \cdot X(2))+(p c=4 \Rightarrow c \cdot X(1))$.

Also, $X_{1}^{\prime \prime}\left(d^{\prime}, e^{0}\right) \approx X\left(1, d^{\prime}, e^{0}\right)$ where $X(p c:\{1,2\}, d:$ $D, e: D)=\left(\sum_{e: D} p c=1 \Rightarrow a(d+e) \cdot X\left(2, d^{\prime}, e\right)\right)+(p c=$ $\left.2 \Rightarrow c(e) \cdot X\left(1,5, e^{0}\right)\right)$. 


$$
\begin{aligned}
V(\boldsymbol{g}: \boldsymbol{G}) & =\sum_{i \in I} \sum_{\boldsymbol{d}_{i}: \boldsymbol{D}_{\boldsymbol{i}}} c_{i} \Rightarrow a_{i}^{\prime \prime}\left(b_{i}\right) \sum_{\boldsymbol{e}_{i}: \boldsymbol{E}_{\boldsymbol{i}}} f_{i}: V\left(\boldsymbol{n}_{\boldsymbol{i}}\right), \\
W(\boldsymbol{g}: \boldsymbol{G}) & =\sum_{i \in I^{\prime}} \sum_{\boldsymbol{d}_{\boldsymbol{i}}: \boldsymbol{D}_{\boldsymbol{i}}} c_{i} \Rightarrow a_{i}\left(b_{i}\right) \sum_{\boldsymbol{e}_{\boldsymbol{i}}: \boldsymbol{E}_{\boldsymbol{i}}} f_{i}: W\left(\boldsymbol{n}_{\boldsymbol{i}}\right),
\end{aligned}
$$

where

$$
\begin{array}{ll}
a_{i}^{\prime}= \begin{cases}\tau & \text { if } a_{i} \in H \\
a_{i} & \text { otherwise }\end{cases} \\
a_{i}^{\prime \prime}=R\left(a_{i}\right) \quad, \quad b_{i}^{\prime}= \begin{cases}{[]} & \text { if } a_{i} \in H \\
b_{i} & \text { otherwise }\end{cases} \\
\text { other } & , I^{\prime}=\left\{i \in I \mid a_{i} \notin E\right\} .
\end{array}
$$

Proposition 4. For all $\boldsymbol{v} \in \boldsymbol{G}, U(\boldsymbol{v}) \equiv \tau_{H}(X(\boldsymbol{v}))$, $V(\boldsymbol{v}) \equiv \rho_{R}(X(\boldsymbol{v}))$, and $W(\boldsymbol{v}) \equiv \partial_{E}(X(\boldsymbol{v}))$.

\section{IMPLEMENTATION AND CASE STUDY}

We developed a Haskell implementation of all procedures for linearisation of prCRL specifications, parallel composition, hiding, encapsulation and renaming ${ }^{1}$. As Haskell is a functional language, the implementations are almost identical to their mathematical representations in this paper. To test the correctness of the procedures, we used the implementation to linearise all examples in this paper, and indeed found exactly the LPPEs we expected.

To illustrate the possible reductions for LPPEs, we model a protocol, inspired by the various leader election protocols that can be found in literature (i.e., Itai-Rodeh [30]), in prCRL. On this model we apply one reduction manually, and several more automatically. Future work will focus on defining and studying more reductions in detail.

We consider a system consisting of two nodes, deciding on a leader by rolling two dies and comparing the results. When both roll the same number, the experiment is repeated. Otherwise, the node that rolled highest wins. The system can be modelled by the prCRL specification shown in Figure 2. We assume that Die is a data type consisting of the numbers from 1 to 6 , and that $I d$ it a data type consisting of the identifiers one and two. The function other is assumed to provide the identifier different from its argument.

Each component has been given an identifier for reference during communication, and consists of a passive thread $P$ and an active thread $A$. The passive thread waits to receive what the other component has rolled, and then provides the active thread an opportunity to obtain this result. The active thread first rolls a die, and sends the result to the other component (communicating via the comm action). Then it tries to read the result of the other component through the passive process (or blocks until this result has been received). Based on the results, either the processes start over, or they declare their victory or loss.

Linearising this specification we obtain a process with 18 parameters and 14 summands, shown in [27]. Computing the state space we obtain 3763 states and 6158 transitions. Due to the uniform linear format, we can apply several classical

\footnotetext{
${ }^{1}$ The implementation can be found at http://fmt.cs.utwente.nl/tools/prcrl.
}

$$
\begin{aligned}
& P(\text { id }: I d, \text { val }: \text { Die, set }: \text { Bool })= \\
& \text { set } \left.=\text { false } \Rightarrow \sum_{d: \text { Die }} \text { receive }(i d, \text { other }(i d), d) \cdot P(\text { id }, d, \text { true })\right) \\
& + \text { set }=\text { true } \Rightarrow \operatorname{getVal}(\text { val }) \cdot P(\text { id }, \text { val, false }) \\
& A(i d: I d)= \\
& \operatorname{roll}(i d) \sum_{d: D i e} \frac{1}{6}: \operatorname{send}(\text { other }(i d), i d, d) \cdot \sum_{e: D i e} \operatorname{readVal}(e) . \\
& (\quad(d=e \Rightarrow A(i d)) \\
& +(d>e \Rightarrow \text { leader }(i d) \cdot A(i d)) \\
& +(e>d \Rightarrow \text { follower }(i d) \cdot A(i d))) \\
& C(\text { id }: I d)=\partial_{\text {getVal,readVal }}(P(\text { id }, 1, \text { false }) \| A(\text { id })) \\
& S=\partial_{\text {send,receive }}(C(\text { one }) \| C(\text { two })) \\
& \gamma(\text { receive }, \text { send })=\text { comm } \\
& \gamma(\text { getVal }, \text { readVal })=\text { checkVal }
\end{aligned}
$$

Figure 2. A prCRL model of a leader election protocol.

reduction techniques to the result. Here we will demonstrate the applicability of four such techniques using one of the summands as an example:

$$
\begin{aligned}
& \sum_{e 21: D i e} p c 21=3 \wedge p c 11=1 \wedge \text { set } 11 \wedge \text { val11 }=e 21 \Rightarrow \\
& \text { checkVal }(\text { val1 } 1) \sum_{(k 1, k 2):\{*\} \times\{*\}} \text { multiply }(1.0,1.0): \\
& Z(1, \text { id11 }, \text { val1 } 1, \text { false }, 1,4, i d 21, d 21, e 21, \\
& \quad \text { c12, id12, val12, set } 12, d 12, p c 22, i d 22, d 22, e 22)
\end{aligned}
$$

Constant elimination [19]. Syntactic analysis of the LPPE revealed that $p c 11, p c 12, i d 11, i d 12, i d 21, i d 22, d 11$ and $d 12$ never get any value other than their initial value. Therefore, these parameters can be removed and everywhere they occur their initial value is substituted for them.

Summation elimination [16]. The summand at hand ranges $e 21$ over Die, but the condition requires it to be equal to val11. Therefore, the summation can be removed and occurrences of $e 21$ substituted by vall1. This way, all summations of the LPPE can be removed.

Data evaluation / syntactic clean-up. After constant elimination, the condition $p c l l=1$ has become $1=1$ and can therefore be eliminated. Also, the multiplication can be evaluated to 1.0, and the Cartesian product can be simplified.

Liveness analysis [20]. Using the methods of [20] we found that after executing the summand at hand vall1 is always first reset before used again. Therefore, we can also immediately reset it after this summand, thereby reducing the state space. This way, two resets have been added.

Combining all these methods to the complete LPPE (the first three automatically, the last one manually), a strongly probabilistic bisimilar LPPE was obtained (see [27] for the details). The summand discussed above became:

$$
\begin{aligned}
& p c 21=3 \wedge \text { set } 11 \Rightarrow \operatorname{checkVal}(\operatorname{val11}) \sum_{k:\{*\}} 1.0: \\
& Z(1, \text { false }, 4, d 21, \operatorname{val11}, \operatorname{val12}, \operatorname{set} 12, p c 22, d 22, e 22)
\end{aligned}
$$

Computing the state space of the reduced LPPE we obtained 1693 states $(-55 \%)$ and 2438 transitions (-60\%). 


\section{CONCLUSIONS AND FUTURE WORK}

This paper introduced a linear process algebraic format for systems incorporating both nondeterministic and probabilistic choice. The key ingredients are: (1) the combined treatment of data and data-dependent probabilistic choice in a fully symbolic manner; (2) a symbolic transformation of probabilistic process algebra terms with data into this linear format, while preserving strong probabilistic bisimulation. The linearisation is the first essential step towards the symbolic minimisation of probabilistic state spaces, as well as the analysis of parameterised probabilistic protocols. The results show that the treatment of probabilities is simple and elegant, and rather orthogonal to the traditional setting [16]. Future work will concentrate on automating the translation from LPPE to PA, and on branching bisimulation preserving symbolic minimisation techniques such as confluence reduction [26] — techniques already proven useful for LPEs.

\section{REFERENCES}

[1] J. Groote and A. Ponse, "The syntax and semantics of $\mu$ CRL," in Proc. of Algebra of Communicating Processes, ser. Workshops in Computing, 1995, pp. 26-62.

[2] http://www.prismmodelchecker.org/.

[3] C. Baier, F. Ciesinski, and M. Größer, "PROBMELA: a modeling language for communicating probabilistic processes," in Proc. of the 2nd ACM/IEEE Int. Conf. on Formal Methods and Models for Co-Design (MEMOCODE), 2004, pp. 57-66.

[4] H. Bohnenkamp, P. D'Argenio, H. Hermanns, and J.-P. Katoen, "MODEST: A compositional modeling formalism for hard and softly timed systems," IEEE Transactions of Software Engineering, vol. 32, no. 10, pp. 812-830, 2006.

[5] P. D'Argenio, B. Jeannet, H. Jensen, and K. Larsen, "Reachability analysis of probabilistic systems by successive refinements," in Proc. of the Joint Int. Workshop on Process Algebra and Probabilistic Methods, Performance Modeling and Verification (PAPM-PROBMIV), ser. LNCS, vol. 2165, 2001, pp. 39-56.

[6] L. de Alfaro and P. Roy, "Magnifying-lens abstraction for Markov decision processes," in Proc. of the 19th Int. Conf. on Computer Aided Verification (CAV), ser. LNCS, vol. 4590, 2007, pp. 325-338.

[7] T. Henzinger, M. Mateescu, and V. Wolf, "Sliding window abstraction for infinite Markov chains," in Proc. of the 21st Int. Conf. on Computer Aided Verification (CAV), ser. LNCS, vol. 5643, 2009, pp. 337-352.

[8] J.-P. Katoen, D. Klink, M. Leucker, and V. Wolf, "Threevalued abstraction for continuous-time Markov chains," in Proc. of the 19th Int. Conf. on Computer Aided Verification (CAV), ser. LNCS, vol. 4590, 2007, pp. 311-324.

[9] M. Kwiatkowska, G. Norman, and D. Parker, "Game-based abstraction for Markov decision processes," in Proc. of the 3rd Int. Conf. on Quantitative Evaluation of Systems (QEST), 2006, pp. 157-166.

[10] H. Hermanns, B. Wachter, and L. Zhang, "Probabilistic CEGAR," in Proc. of the 20th Int. Conf. on Computer Aided Verification (CAV), ser. LNCS, vol. 5123, 2008, pp. 162-175.

[11] M. Kattenbelt, M. Kwiatkowska, G. Norman, and D. Parker, "Abstraction refinement for probabilistic software," in Proc. of the 19th Int. Conf. on Verification, Model Checking, and Abstract Interpretation (VMCAI), ser. LNCS, vol. 5403, 2009, pp. 182-197.
[12] J. Hurd, A. McIver, and C. Morgan, "Probabilistic guarded commands mechanized in HOL," Theoretical Computer Science, vol. 346, no. 1, pp. 96-112, 2005.

[13] M. Bezem and J. Groote, "Invariants in process algebra with data," in Proc. of the 5th Int. Conf. on Concurrency Theory (CONCUR), ser. LNCS, vol. 836, 1994, pp. 401-416.

[14] K. Larsen and A. Skou, "Bisimulation through probabilistic testing," Information and Computation, vol. 94, no. 1, pp. $1-28,1991$.

[15] D. Bosscher and A. Ponse, "Translating a process algebra with symbolic data values to linear format," in Proc. of the 1st Workshop on Tools and Algorithms for the Construction and Analysis of Systems (TACAS), ser. BRICS Notes Series, vol. NS-95-2, 1995, pp. 119-130.

[16] Y. Usenko, "Linearization in $\mu$ CRL," Ph.D. dissertation, Eindhoven University of Technology, 2002.

[17] J. Groote and J. Springintveld, "Focus points and convergent process operators: a proof strategy for protocol verification," Journal of Logic and Algebraic Programming, vol. 49, no. 1-2, pp. 31-60, 2001.

[18] W. Fokkink, J. Pang, and J. van de Pol, "Cones and foci: A mechanical framework for protocol verification," Formal Methods in System Design, vol. 29, no. 1, pp. 1-31, 2006.

[19] J. Groote and B. Lisser, "Computer assisted manipulation of algebraic process specifications," CWI, Tech. Rep. SENR0117, 2001.

[20] J. van de Pol and M. Timmer, "State space reduction of linear processes using control flow reconstruction," in Proc. of the 7th Int. Symp. on Automated Technology for Verification and Analysis (ATVA), ser. LNCS, vol. 5799, 2009, pp. 54-68.

[21] M. Espada and J. van de Pol, "An abstract interpretation toolkit for $\mu$ CRL," Formal Methods in System Design, vol. 30, no. 3, pp. 249-273, 2007.

[22] S. Blom, B. Lisser, J. van de Pol, and M. Weber, "A database approach to distributed state-space generation," Journal of Logic and Computation, 2009, Advance Access, March 5.

[23] S. Blom and J. van de Pol, "Symbolic reachability for process algebras with recursive data types," in Proc. of the 5th Int. Colloquium on Theoretical Aspects of Computing (ICTAC), ser. LNCS, vol. 5160, 2008, pp. 81-95.

[24] J. Groote and R. Mateescu, "Verification of temporal properties of processes in a setting with data," in Proc. of the 7th Int. Conf. on Algebraic Methodology and Software Technology (AMAST), ser. LNCS, vol. 1548, 1998, pp. 74-90.

[25] J. Groote and T. Willemse, "Model-checking processes with data," Science of Computer Programming, vol. 56, no. 3, pp. 251-273, 2005.

[26] S. Blom and J. van de Pol, "State space reduction by proving confluence," in Proc. of the 14th Int. Conf. on Computer Aided Verification (CAV), ser. LNCS, vol. 2404, 2002, pp. 596-609.

[27] J.-P. Katoen, J. van de Pol, M. Stoelinga, and M. Timmer, "A linear process algebraic format for probabilistic systems with data (extended version)," TR-CTIT-10-??, CTIT, University of Twente, Tech. Rep., 2010.

[28] R. Segala, "Modeling and verification of randomized distributed real-time systems," Ph.D. dissertation, Massachusetts Institute of Technology, 1995.

[29] R. Milner, Communication and Concurrency. Prentice-Hall, 1989.

[30] W. Fokkink and J. Pang, "Variations on Itai-Rodeh leader election for anonymous rings and their analysis in PRISM," Journal of Universal Computer Science, vol. 12, no. 8, pp. 981-1006, 2006. 